\title{
Catheter Biopsy as a Useful Tool to Establish an Early Diagnosis for Canine Prostatic Disorders
}

\author{
K. PACLÍKOVÁ, P. KOHOUT, M. VLAŠÍN
}

Small Animal Clinic, Faculty of Veterinary Medicine, University of Veterinary and Pharmaceutical Sciences Brno, Czech Republic

Received October 2, 2006

Accepted July 9, 2007

\begin{abstract}
Paclíková K., P. Kohout, M. Vlašín: Catheter Biopsy as a Useful Tool to Establish an Early Diagnosis for Canine Prostatic Disorders. Acta Vet. Brno 2007, 76: 475-485.

In the study conducted on 29 pre-selected patients, we intended to evaluate the reproducibility of prostatic catheter biopsy (CB) as a minimally invasive procedure, directly comparing it to another well-established method, namely ultrasound-guided fine-needle aspiration biopsy (USG-FNAB). As a direct control we used histopathological evaluation and microbial cultivation of samples obtained by percutaneous Tru-Cut biopsy. Evaluating our data, we found that for establishing proper diagnosis by means of cytology, less invasive catheter biopsy (CB 75.86\%) is at least comparable to already proven ultrasound-guided fine needle aspiration biopsy (USG-FNAB 72.41\%), while catheter biopsy (CB 13 cases out of 29) was significantly $(p<0.0001)$ more sensitive in actual identification of infectious agents than ultrasound-guided fine needle aspiration biopsy (USG-FNAB 2 cases of 29). We believe that an early microbial evaluation of patients suffering from prostatic disorders helps to target antimicrobial treatment in the beginning of therapy, reducing the risk of failure due to bacterial resistance.
\end{abstract}

Dog, urology, prostate gland, FNAB, prostatic massage, prostatic fluid cultivation

The prostate gland, the only accessory sex gland in male dog is located adjacent to the bladder neck in the pelvic inlet. It is a two-lobular gland with fibrous septum in the middle. Limitation of this septum is in the dorsal sulcus, mostly distinct during rectal palpation (Barsanti and Finco 1989). Its major function is secretion of the prostatic fluid, which is part of the first and third fraction of ejaculate (England et al. 1990). Various prostatic disorders are common in old intact dogs (Barsanti and Finco 1989; Krawiec 1994), as well as in men. Symptomatology of the prostatic disease can be generally found in dogs older than 5.5 years (Krawiec and Heflin 1992). Well-described disorders include benign prostatic hyperplasia (BPH), squamous metaplasia, prostatic inflammation, both acute and chronic, inflammation-related prostatic abscedation, (with single or multiple abscesses), prostatic cysts and prostatic neoplasia.

Prostatic catheter biopsy is quite a simple method, feasible even in fractious dogs or patients in pain or severe discomfort, when manual ejaculation (collection of third fraction) is not possible (Barsanti and Finco 1989). This method stands on the borderline between invasive and non-invasive methods of examination and it is generally advisable to clients refusing percutaneous biopsy techniques. Fluid obtained by catheter biopsy can be submitted to both cytology and bacterial cultivation.

The goal of our study was to evaluate sensitivity of prostatic catheter biopsy as a method in clinical practice partially forgotten and to express its importance in the management of canine prostatic disorders.

\section{Materials and Methods}

Animals

Twenty nine male dogs, patients of Veterinary teaching hospital, University of Veterinary and Pharmaceutical Sciences Brno, Czech Republic, were included into the study. The patients were presented between October

Address for correspondence:

K. Paclíková

Faculty of Veterinary Medicine

University of Veterinary and Pharmaceutical Sciences

Brno, Czech Republic

Phone: +420 541562309

E-mail: paclikovak@vfu.cz

http://www.vfu.cz/acta-vet/actavet.htm 
2004 and June 2006, with various prostatic symptomatologies, with the age between 4 and 13 years, weighing between $1.5 \mathrm{~kg}$ and $80 \mathrm{~kg}$. The most often observed symptoms included: tenesmus, haematuria, urethral discharge, recurrent low-urinary tract infection, dysuria, perineal herniation, lethargy, vomitus and caudal abdominal pain. Some of the patients were referred with a prostatic disease by a local veterinarian.

\section{Inclusion criteria}

For each patient we obtained detailed history (previous and recent problems, diseases and accidents, related to the urinary tract) and complete physical examination was performed accordingly. Specific examination of the urinary tract along with prostatic palpation always followed. The evaluation of the urinary tract included routine urinalysis with specific gravity analysis and urinary sediment examination after aseptic cystocentesis. Then, routine ultrasonography of the urinary tract followed (Pro Sound 5000 SSD, Aloka Co., LTD, Japan). After that, rectal palpation was performed with the help of caudodorsal abdominal pressure. Rectal palpation was evaluated according to Krawiec (1994); data are presented in the Table 1.

Table 1. Diagnostic values for rectal palpation of the prostate gland according to Krawiec (1994)

\begin{tabular}{|l|l|}
\hline Prostatic disease & Rectal palpation \\
\hline Bacterial prostatitis & $\begin{array}{l}\text { Symmetric, apparently painful (acute prostatitis) } \\
\text { Symmetric, with no signs of pain (chronic prostatitis) }\end{array}$ \\
\hline Prostatic cysts & $\begin{array}{l}\text { Symmetric, solid and enlarged, small fluctuations } \\
\text { In case of big cyst, apparent asymmetry with fluctuation }\end{array}$ \\
\hline Neoplasia & Asymmetric, solid and enlarged, not mobile \\
\hline Benign prostatic hyperplasia & Symmetric, solid, enlarged, no sign signs of pain \\
\hline Paraprostatic cysts & Asymmetric fluctuation in the close vicinity of prostate \\
\hline Prostatic abscess & Asymmetric enlargement, solid or with fluctuation \\
\hline
\end{tabular}

Generally, patients included in the study showed clinically manifest prostatic disorders according to history and physical examination, with significant changes revealed by rectal palpation, with or without positive urinalysis.

Sample obtaining

All samples were collected after 24-hour period of fasting, from sedated patients (medetomidine $10 \mu \mathrm{g} / \mathrm{kg}$, butorphanol $1 \mathrm{mg} / \mathrm{kg}$, both i.v.). Whenever needed, general anaesthesia was introduced using propofol $1 \mathrm{mg} / \mathrm{kg}$ i.v.. Then we performed USG-guided aseptic cystocentesis, prostatic catheter biopsy, USG-FNAB, and finally Tru-Cut percutaneous biopsy, proceeding from the least invasive method to the most aggressive one. The patient positioning was always in dorsal or dorso-lateral recumbence.

Catheter biopsy

Preputium was repeatedly flushed with sterile saline solution. Then, aseptic catheterisation (sterile canine urinary catheter, lubricated by mesocain gel) of the urinary bladder was performed. All urine was meticulously removed and bladder was flushed by sterile saline solution. Catheter was partially withdrawn under the control of rectal digital palpation and left close to the caudal border of prostatic urethra. Afterwards, digital massage of the prostate gland was carefully performed (about 2 minutes). Whenever prostate could not be reached rectally, caudal abdominal massage was performed instead, while the position of the catheter was controlled by ultrasonography. Approximately 1 minute after commencing of massage, negative pressure was gently applied by an assistant, using a 10cc syringe. Moving the catheter back and forth (with the gap of about $10 \mathrm{~mm}$ ) facilitated fluid collection. After collecting prostatic fluid, syringe was disconnected and catheter was left in place for further procedures.

Ultrasound-guided fine needle aspiration biopsy. Parapreputial region was clipped and aseptically scrubbed. Then, $22 \mathrm{G}$ needle put on a 20cc syringe was introduced under USG control deep into prostatic glandular tissue, negative pressure through the syringe was applied and the needle was manipulated within the gland repeatedly. After releasing negative pressure, the needle was withdrawn and its content was pushed out to the slide, as well as to the cultivation media (Amies).

Sample evaluation

The basic data we obtained from a brief analysis of Hepta-phan strips $(\mathrm{pH}$, total protein concentration, glucose, ketones, urobilinogen, bilirubin and traces of blood) were interpreted in the scale from 0 to ++++ and recorded in the protocol of each patient. The other examinations were performed as usual.

The size of the prostate gland was determined by ultrasonography according to Ruel et al. (1997): $\mathrm{V}_{1}\left(\mathrm{~cm}^{3}\right)=$ $(0.867 \times$ body weight $)+(1.885 \times$ age $)$

$\mathrm{V}_{2}\left(\mathrm{~cm}^{3}\right)=$ length $(\mathrm{cm}) \times$ height $(\mathrm{cm}) \times$ width $(\mathrm{cm}) \times 0.523$.

$\mathrm{V}_{1}\left(\mathrm{~cm}^{3}\right)$ was compared with $\mathrm{V}_{2}\left(\mathrm{~cm}^{3}\right)$ (Table 4$)$.

Then position, echogenicity shape and surface were evaluated (Feeney et al. 1987; Lamb 1990; Bell et al. 1991; Lüerssen 1993; Prüfer et al. 2000). 
Cytology of samples

Cytology was performed blindly on samples obtained from both Catheter biopsy and USG-guided fine needle aspiration biopsy without any knowledge of histopathology (Bell et al. 1991; Dorfman and Barsanti 1995; Peter et al. 1995; Baker and Lumsden 1999). Moreover, we established grading system for severity of inflammation during examination of the urine sediment in $\times 400$ magnification using the light microscope (Table 3). Inflammation was considered acute whenever neutrophils reached at least $70 \%$ of all inflammatory cells, whereas it was considered chronic whenever macrophages reached the borderline of 50\% of all cells (Rebar 1987). Differentiations between cysts and abscesses were performed based on cytology of the aspirate.

Histopathology of the prostate

Histopathology of each bioptate was performed at the Department of Pathology, Faculty of Veterinary Medicine, and then evaluated according to Atilola and Pennock (1986), Lowseth (1990), Nielsen and Kennedy (1990), and Bell et al. (1991). It was considered confirmative for evaluation of each less-invasive diagnostic procedure (USG-FNAB and catheter biopsy).

Tissue cultivation

Any bacterial growth identified from urine after cystocentesis or FNAB sample was considered positive, while only more than $10^{3}$ of bacteria in $1 \mathrm{ml}$ were regarded for prostatic catheter biopsy (Barsanti and Finco 1989).

\section{Statistical analysis}

The results were put into tables to be analyzed statistically using McNemara's test. On specific occasions, the Positive correlation test was applied and $p$ value was set at 0.05 for significance and at 0.01 for high significance. Basic ratio was often expressed in per cent $(\%)$.

\section{Results}

Among the problems most frequently reported by owners, tenesmus was predominant, $(\mathrm{n}=21$, in 3 patients as the only symptom), while the others were: lethargy $(\mathrm{n}=10)$, haematuria $(n=10)$ bloody discharge from penis, not during urination $(n=10)$, vomitus $(n=9)$, caudal abdominal pain $(n=7)$, dysuria $(n=5)$, bloody-purulent discharge $(n=5)$, recurrent uroinfection $(n=4)$, fever $(n=4)$ and purulent only discharge from the penis $(n$ $=1$ ). Typically, these symptoms occurred in various combinations, very rarely as a single trouble (Table 1).

\section{Urinalysis}

Urine was found negative in 7 patients; traces of blood were found in 17 patients (in 5 of them as the only finding); proteinuria was discovered in 16 patients (in 5 of them as the only finding). A combination of microscopic haematuria and proteinuria was found in 11 patients (and in 5 without any other finding). Alkaline $\mathrm{pH}$ was found in 7 dogs, while in 1 patient a combination of alkaline $\mathrm{pH}$ and haematuria and in 6 of them a combination with proteinuria was found. The urine sediment evaluation revealed similar data, with 7 dogs negative and 11 cases with typical moderate and 4 severe inflammatory changes (Tables 3 and 5).

\section{Palpation of the prostate gland}

Based on criteria set up by Krawiec (1994) (Table 2), after rectal palpation, the preliminary diagnosis of BPH was established in 19 patients, even though histopathology revealed BPH in only 12 cases. In this case, the difference of false positive results was statistically significant $(p<0.05)$. Chronic prostatitis was discovered by palpation in 4 patients only, while histopathologically the chronic inflammation was confirmed in 16 cases. This difference was statistically highly significant for false negative results $(p<$ 0.0006). In 4 patients we discovered acute prostatitis, while in 11 patients fluctuations (cysts or abscesses) were found: 6 in combination with BPH, 1 along with chronic and 3 along with acute prostatitis (Table 5).

\section{Ultrasonography}

By means of ultrasonography, we discovered prostatic enlargement in 28 out of 29 patients, while in one case the size of the gland was decreased (see Table 4 and 5). Based 
Table 2. Historical data obtained from 29 patients included into the study

\begin{tabular}{|c|c|c|c|c|c|c|c|c|c|c|c|}
\hline No. & Breed & vomit & $\mathrm{F}$ & Let & AP & TEN & DYS & RUI & $\mathrm{HU}$ & $\begin{array}{c}\text { Discharge } \\
\text { B/P }\end{array}$ & Other \\
\hline 1 & Crossbred & - & - & - & - & + & - & - & - & $-/-$ & \\
\hline 2 & Cocker Spaniel & - & - & - & - & + & - & - & - & $-/-$ & \\
\hline 3 & German Shepherd & + & - & - & - & + & - & - & - & $-/-$ & Perineal hernia \\
\hline 4 & Springer Spaniel & - & - & + & - & + & - & - & - & $-/-$ & \\
\hline 5 & Sarplaninac & - & - & - & - & - & - & - & + & $+/ t$ & Cryptorchism \\
\hline 6 & Great Dane & + & - & + & + & - & + & - & - & $-/-$ & \\
\hline 7 & Standard Schnauzer & + & + & + & - & + & - & - & - & $-/-$ & \\
\hline 8 & Cocker Spaniel & + & - & + & - & + & - & - & - & $-/-$ & \\
\hline 9 & Crossbred & - & - & - & + & - & - & - & + & $+/-$ & \\
\hline 10 & Crossbred & - & - & - & + & + & - & - & + & $+/ t$ & Car accident a year ago \\
\hline 11 & German Shepherd & - & + & + & - & + & - & - & - & $-/-$ & Local alopecia \\
\hline 12 & German Shepherd & - & - & - & - & - & - & + & - & $+/-$ & Epilepsy \\
\hline 13 & Venden Basset & - & - & - & - & + & - & - & - & $-/+$ & \\
\hline 14 & Crossbred & - & - & - & + & - & + & - & - & $+/ t$ & Tracheal collapse \\
\hline 15 & Papillon & + & + & + & + & + & - & - & - & $-/-$ & \\
\hline 16 & Greater Swiss Mountain Dog & - & - & - & - & + & + & - & - & $-/-$ & Perineal hernia \\
\hline 17 & German Shepherd & + & - & + & - & + & - & - & + & $+/-$ & Cachexia \\
\hline 18 & Golden Retriever & - & - & - & - & - & - & + & + & $+/-$ & \\
\hline 19 & Bernese Mountain Dog & - & - & - & - & - & - & + & + & $+/-$ & \\
\hline 20 & Miniature Schnauzer & & - & + & + & + & - & - & - & $+/-$ & Cryptorchism \\
\hline 21 & German Shepherd & + & + & + & + & + & + & + & + & $+/-$ & Cachexia, hypotrichosis \\
\hline 22 & German Shepherd & - & - & - & - & + & - & - & + & $-/-$ & \\
\hline 23 & Pitbullterrier & + & + & + & - & - & - & - & - & $-/-$ & \\
\hline 24 & Pomeranian & - & - & - & - & + & - & - & - & $-/-$ & vestibular syndrome \\
\hline 25 & Dachshund & - & - & - & - & + & - & - & - & $+/-$ & \\
\hline 26 & Pitbullterrier & - & - & - & - & + & - & - & - & $+/+$ & \\
\hline 27 & Dachshund & + & - & - & - & + & + & - & + & $+/+$ & \\
\hline 28 & German Shepherd & - & - & - & - & + & - & - & - & $+/-$ & Perineal hernia \\
\hline 29 & Airedale Terrier & - & - & - & - & + & - & - & + & $+/-$ & \\
\hline
\end{tabular}

AP-abdominal pain, Let-lethargy, DYS-dysuria, F-fever, HU-hematuria, Discharge-Bloody/Purulent RUI-repeated uroinfect, TEN-tenesmus, vomit-vomitus

on literature (Feeney et al. 1987; Lamb 1990; Bell et al. 1991; Lüerssen 1993; Prüfer et al. 2000), we attempted to establish the diagnosis of BPH in 14 cases, chronic prostatitis in 12 patients, while cysts or abscesses were observed in 18 cases (Tables 4 and 5).

The diagnosis was based on histopathology and cytology after catheter biopsy and (USGguided) fine needle aspiration biopsy.

Histopathology revealed $\mathrm{BPH}$ in 12 dogs, chronic prostatitis in 16 dogs and acute prostatitis in $1 \mathrm{dog}$. There were some other histopathological changes, not necessarily related to the final diagnosis. These changes are listed in Table 6. For transparency, we show relevant data in parentheses for each diagnostic procedure of the scope.

Based on prostatic catheter biopsy we diagnosed BPH in 16 cases (12 based on histopathology), chronic prostatitis in 9 cases (16 based on histopathology) and acute prostatitis in 4 patients (1 based on histopathology). For chronic prostatitis it means 7 false negative cases, which is statistically highly significant difference compared to histopathology $(p<0.009)$. For acute prostatitis, we found insufficient number of patients to make statistical conclusions, so we can only postulate that 1 false positive case of acute 
Table 3. Grading of prostatic inflammation according to urinalysis

\begin{tabular}{|l|c|c|c|c|}
\hline Urine sediment & $\begin{array}{c}\text { Leukocytes } \\
\text { (number of } \\
\text { cells in view) }\end{array}$ & $\begin{array}{c}\text { Erythrocytes } \\
\text { (number of } \\
\text { cells in view) }\end{array}$ & $\begin{array}{c}\text { Epithelial cells } \\
\text { - transitional } \\
\text { (number of } \\
\text { cells in view) }\end{array}$ & $\begin{array}{c}\text { Bacteria } \\
\text { (F/NF) }\end{array}$ \\
\hline Intact & $0-4$ & $0-4$ & 0 & 0 \\
\hline Erythrocytes only & 0 & sporadic/overload & 0 & 0 \\
\hline Mild inflammation & $5-10$ & $5-15$ & sporadic & sporadic \\
\hline Strong inflammation & overload & sporadic/overload & massive & massive \\
\hline
\end{tabular}

Magnification $\times 400$, Bacteria F/NF-fagocytated/non-fagocytated, Massive-cells in bigger convolutes, covering almost entire view, Sporadic-individual cells, occasionally in small groups

Overload-cells covering entire view, often touching or overlapping each other

Table 4. Size of the prostate gland according to Ruel et al. (1997)

\begin{tabular}{|l|l|}
\hline $\mathrm{V}^{1}>\mathrm{V}^{2}$ & Attenuation \\
\hline $\mathrm{V}^{1}=\mathrm{V}^{2}$ & Normal size \\
\hline $\mathrm{V}^{1}<\mathrm{V}^{2}$ & Enlargement \\
\hline
\end{tabular}

inflammation was recorded during our study.

Based on USG-guided fine needle aspiration biopsy we diagnosed 16 patients as suffering from BPH (12 based on histopathology), 10 patients having chronic prostatitis (16 based on histopathology), and 3 suffering from acute inflammation (same as by histopathology). Again, a high number of false negative cases of chronic inflammation were found. Six patients make a statistically significant difference $(p<0.05)$ compared to histopathology (see Table 6).

Comparsion of rectal palpation and ultrasonography with cytology (after USG-FNAB or $\mathrm{CB}$ ) and histopatology

After rectal palpation, the same results as after catheter biopsy $(\mathrm{CB})$ were found in $62.04 \%(\mathrm{n}=18)$, whereas the same results as after USG-FNAB were found in $55.17 \%$ of cases $(n=15)$. Correlation between rectal palpation and confirmative histopathology was in $51.72 \%(\mathrm{n}=15)$.

Ultrasonography suggested the same diagnosis as all the other methods in $44.82 \%(\mathrm{n}=$ 13) (Table 6).

Generally, prostatic Catheter biopsy revealed the same results as confirmative histopathology in $75.86 \%(\mathrm{n}=22)$, while USG-guided Fine needle aspiration biopsy revealed the same results as histopathology in $72.41 \%(\mathrm{n}=21)$. With that regard, the difference between groups was not statistically significant.

\section{Bacterial cultivation}

Cultivation of prostatic fluid obtained from catheter biopsy appeared positive in 13 cases, while cultivation of fine needle aspirate gave only 2 positive results (statistically highly significant difference, $p<0.0001$ ), and urine cultivation revealed 6 positive results only. Of these 13 positive patients, 8 suffered from chronic inflammation, confirmed by histopathology, whereas in 4 cases, BPH was the reason and in 1 case, acute prostatitis was revealed. Cultivation of aspirate from cysts or abscesses gave 1 positive result out of 9. When we applied our grading system of inflammation (Table 2), we found that $100 \%$ of the sediment with "strong inflammation" revealed a pathogen by cultivation. On the other hand, we found all samples of "intact" sediment $(\mathrm{n}=7)$, as well as samples containing "erythrocytes only" ( $\mathrm{n}=7)$, negative (no pathogen was identified after cultivation). In cultivation of the sediment revealing mild inflammation, only 3 samples of $11(27.27 \%)$ revealed any pathogen.

Altogether, using cultivation we identified 4 pathogens: Pseudomonas aeruginosa (46.15\% - prostatic fluid; 0 in urine), Escherichia coli (30.7\% - prostatic fluid; $33.33 \%$ - urine), Streptococcus sp. (15.38\% - prostatic fluid; 33.33\% - urine) and Klebsiella 
Table 5. Preliminary diagnosis established after urinalysis, rectal palpation and ultrasonography

\begin{tabular}{|c|c|c|c|c|c|}
\hline No. & Heptaphan & Sediment & Rectal palpation & USG & PS \\
\hline 1 & neg. & Intact & $\mathrm{CHP}, \mathrm{c} / \mathrm{a}$ & A, CHP & EN \\
\hline 2 & neg. & Intact & $\mathrm{BPH}$ & $\mathrm{BPH}, \mathrm{SC}$ & EN \\
\hline 3 & $\mathrm{P}+, \mathrm{H}++$ & Mild inflammatory & $\mathrm{BPH}, \mathrm{c} / \mathrm{a}$ & $\mathrm{CHP}$ & EN \\
\hline 4 & $\begin{array}{l}\mathrm{pH} 8, \\
\mathrm{P}++, \mathrm{H}++\end{array}$ & Mild inflammatory & $\mathrm{BPH}$ & IC, BPH, PARAC & EN \\
\hline 5 & $\mathrm{H}++++$ & Ery only & $\mathrm{BPH}$ & $\mathrm{IC}, \mathrm{BPH}$ & EN \\
\hline 6 & $\mathrm{P}+, \mathrm{H}+$ & Mild inflammatory & cysts & $\mathrm{IC}, \mathrm{BPH}$ & EN \\
\hline 7 & $\begin{array}{l}\mathrm{pH} 9, \\
\mathrm{P}+++, \mathrm{H}+\end{array}$ & Strong inflammatory & $\mathrm{CHP}$ & $\mathrm{CHP}$ & EN \\
\hline 8 & $\mathrm{pH} 8, \mathrm{H}++$ & Mild inflammatory & $\mathrm{AP}, \mathrm{c} / \mathrm{a}$ & A, CHP & EN \\
\hline 9 & $\mathrm{P}++$ & Mild inflammatory & $\mathrm{BPH}$ & $\mathrm{BPH}$ & EN \\
\hline 10 & $\mathrm{P}+$ & Mild inflammatory & AP & A, CHP & EN \\
\hline 11 & $\begin{array}{l}\mathrm{pH} \mathrm{9,} \\
\mathrm{P}++, \mathrm{H}++\end{array}$ & Strong inflammatory & $\mathrm{BPH}$ & $\mathrm{IC}, \mathrm{BPH}$ & EN \\
\hline 12 & neg. & Intact & $\overline{\mathrm{BPH}}$ & IC, PARAC, BPH & EN \\
\hline 13 & neg. & Intact & $\mathrm{BPH}, \mathrm{c} / \mathrm{a}$ & $\mathrm{IC}, \mathrm{CHP}$ & EN \\
\hline 14 & $\mathrm{H}++$ & Ery only & $\mathrm{BPH}$ & $\mathrm{CHP}$ & EN \\
\hline 15 & $\mathrm{P}+, \mathrm{H}++$ & Mild inflammatory & $\mathrm{AP}, \mathrm{c} / \mathrm{a}$ & $\mathrm{A}$ & EN \\
\hline 16 & neg. & Intact & $\mathrm{BPH}$ & $\mathrm{IC}, \mathrm{BPH}$ & EN \\
\hline 17 & $\mathrm{P}++$ & Mild inflammatory & $\mathrm{BPH}, \mathrm{c} / \mathrm{a}$ & $\mathrm{IC}, \mathrm{BPH}$ & EN \\
\hline 18 & $\begin{array}{l}\mathrm{pH} 8, \mathrm{P}+, \\
\mathrm{H}++\end{array}$ & Mild inflammatory & $\mathrm{BPH}$ & $\mathrm{BPH}$ & EN \\
\hline 19 & $\mathrm{P}++$ & Mild inflammatory & $\mathrm{BPH}$ & $\mathrm{IC}, \mathrm{BPH}$ & EN \\
\hline 20 & $\mathrm{P}++$ & Mild inflammatory & $\mathrm{BPH}$ & $\mathrm{CHP}$ & EN \\
\hline 21 & $\begin{array}{l}\mathrm{pH} \mathrm{9,} \\
\mathrm{P}++++, \mathrm{H}++\end{array}$ & $\begin{array}{l}\text { Strong } \\
\text { inflammatory }\end{array}$ & $\mathrm{AP}, \mathrm{c} / \mathrm{a}$ & $\mathrm{A}, \mathrm{CHP}$ & EN \\
\hline 22 & $\mathrm{H}+++$ & Ery only & $\mathrm{BPH}, \mathrm{c} / \mathrm{a}$ & $\mathrm{IC}, \mathrm{SC}$ & EN \\
\hline 23 & $\mathrm{P}+, \mathrm{H}+++$ & Ery only & $\mathrm{BPH}$ & Mineralizace, CHP & EN \\
\hline 24 & neg. & Intact & $\mathrm{CHP}$ & $\mathrm{IC}, \mathrm{BPH}$ & AT \\
\hline 25 & neg. & Intact & $\mathrm{CHP}$ & $\mathrm{BPH}$ & $\mathrm{EN}$ \\
\hline 26 & $\mathrm{P}+, \mathrm{H}++$ & Ery only & $\mathrm{BPH}$ & $\mathrm{IC}, \mathrm{CHP}$ & EN \\
\hline 27 & $\begin{array}{l}\mathrm{pH} 8, \\
\mathrm{P}++++, \mathrm{H}+\end{array}$ & $\begin{array}{l}\text { Strong } \\
\text { inflammatory }\end{array}$ & AP & AP & EN \\
\hline 28 & $\mathrm{H}++$ & Ery only & $\mathrm{BPH}$ & $\mathrm{BPH}$ & $\mathrm{ZV}$ \\
\hline 29 & $\mathrm{H}+++$ & Ery only & $\mathrm{BPH}$ & $\mathrm{CHP}$ & $\mathrm{ZV}$ \\
\hline
\end{tabular}

Sedimen-grading of inflammation according to table 3, A - abscess, AP - acute prostatitis, BPH - benign prostatic hyperplasia, c/a - cyst/abscess, CHP - chronic prostatitis, IC - intraprostatic cyst, H - Haematuria PARAC - paraprostatic cyst, Ery - erythrocytes, P - proteinuria, SC - subcapsular cyst,

USG - ultrasonography, PS - prostatic size, AT - attenuation, EN - enlargement

pneumoniae (7.96\% - prostatic fluid; $16.66 \%$ - urine). Corynebacterium pyogenes was identified once from the prostatic fluid, however, not in sufficient concentration (Table 7).

\section{Discussion}

The reasons why the owners sought the help of a veterinarian were most frequently tenesmus, followed by lethargy of unknown origin, urethral discharge, haematuria and vomitus. According to Read and Bryden (1995), bloody urethral discharge is the most common symptom, observed as the only irregularity in $72 \%$ patients. In our study, only 6 
Table 6. Prostatic cytology (CB, FNAB) and histopathology

\begin{tabular}{|l|l|l|l|l|l|}
\hline No. & CB & Cyst/abscess & FNAB & HP & *note to HP \\
\hline 1 & CHP & A & BPH & CHP & \\
\hline 2 & BPH & & BPH & BPH & \\
\hline 3 & BPH & C & BPH & BPH & fibrosis \\
\hline 4 & BPH & C & BPH & BPH & fibrosis \\
\hline 5 & BPH & & BPH & BPH & \\
\hline 6 & BPH & & BPH & BPH & \\
\hline 7 & CHP & & CHP & CHP & BPH \\
\hline 8 & AP & A & AP & CHP & \\
\hline 9 & BPH & & CHP & CHP & fibrosis, BPH \\
\hline 10 & CHP & & CHP & CHP & \\
\hline 11 & AP & & AP & CHP & metaplasia \\
\hline 12 & CHP & A & CHP & CHP & BPH \\
\hline 13 & BPH & A & BPH & BPH & metaplasia \\
\hline 14 & BPH & & BPH & BPH & \\
\hline 15 & AP & A & AP & CHP & \\
\hline 16 & BPH & & BPH & BPH & fibrosis \\
\hline 17 & BPH & & BPH & CHP & fibrosis \\
\hline 18 & BPH & & BPH & BPH & \\
\hline 19 & BPH & & BPH & BPH & \\
\hline 20 & BPH & & BPH & BPH & \\
\hline 21 & AP & A & CHP & AP & \\
\hline 22 & BPH & A & CHP & CHP & BPH \\
\hline 23 & BPH & & BPH & CHP & \\
\hline 24 & CHP & & CHP & CHP & fibrosis \\
\hline 25 & CHP & & BPH & CHP & \\
\hline 26 & CHP & & CHP & CHP & \\
\hline 27 & CHP & & CHP & CHP & \\
\hline 28 & CHP & & BPH & BPH & \\
\hline 29 & BPH & & & fibrosis \\
\hline A & & & & & \\
\hline
\end{tabular}

A - abscess, AP - acute prostatitis, BPH - benign prostatic hyperplasia, C - cyst, FNAB - fine needle aspiration biopsy, $\mathrm{CB}$ - catheter biopsy, HP - histopathology, CHP - chronic prostatitis

*note to HP - note to histopathology, other, non-related findings

patients (50\%), with the diagnosis established by histopathology showed originally bloody urethral discharge, however, always in combination with one or more other symptoms. On the other hand, a bit more significant symptom appeared to be tenesmus, observed in 21 patients $(72.24 \%$ ), while in 3 patients as the only clinical sign. The other clinical characteristics, reported among others by Dorfman and Barsanti (1995), we observed in various combinations (Table 1). We agree with other authors (White 2000), that rectal palpation, often with caudal abdominal pressure, is essential in physical examination of patient suspect of prostatic disorder. However, we feel that palpation as a tool for establishing preliminary diagnosis, as reported by Krawiec (1994) does not provide sufficient information, nor does it express enough sensitivity, especially to decide between chronic prostatitis and benign prostatic hyperplasia. In our settings, we found rectal palpation to be dependable only in $51.72 \%$ cases in comparison with histopathology. The difference was statistically highly significant $(p<0.0006)$ for BPH and significant $(p<0.05)$ for chronic inflammation. In our opinion, prostatic rectal palpation can be only explained in the context with other diagnostic tests and procedures. 
Table 7. Bacterial cultivation of urine, prostatic fluid and fine needle aspirate

\begin{tabular}{|c|c|c|c|c|}
\hline No. & Urine & Prostatic fluid & Fine needle aspirate & Abscess/cyst \\
\hline 1 & Neg. & Neg. & Neg. & Neg. (abscess) \\
\hline 2 & $\mathrm{Neg}$ & Corynebacterium sp. $<10^{4}$ & Neg. & \\
\hline 3 & Neg. & Neg. & Neg. & Neg. (cyst) \\
\hline 4 & Neg. & P. aeruginosa $>10^{5}$ & Neg. & Neg. (cyst) \\
\hline 5 & Neg. & Neg. & Neg. & \\
\hline 6 & Neg. & Neg. & Neg. & \\
\hline 7 & E. coli $>10^{5}$ & E. coli $>10^{5}$ & Neg. & \\
\hline 8 & Neg. & Neg. & Neg. & Neg. (abscess) \\
\hline 9 & K. pneumoniae $>10^{3}$ & P. aeruginosa $>10^{5}$ & Neg. & \\
\hline 10 & K. pneumoniae $>10^{3}$, & P. aeruginosa $>10^{5}$ & Neg. & \\
\hline 11 & Str. $\beta$-hemol. sk. G $>10^{5}$ & Str. $\beta$-hemol. sk. G > $10^{5}$ & Str. $\beta$-haemol. sk. G $>10^{5}$ & \\
\hline 12 & Neg. & Neg. & Neg. & Neg. (abscess) \\
\hline 13 & Neg. & P. aeruginosa $>10^{5}$ & Neg. & Neg. (abscess) \\
\hline 14 & Neg. & E.coli $>104$ P. aeruginos $a>10^{5}$ & Neg. & \\
\hline 15 & Neg. & P. aeruginosa $>10^{5}$ & Neg. & Neg. (abscess) \\
\hline 16 & Neg. & Neg. & Neg. & \\
\hline 17 & Neg. & Neg. & Neg. & \\
\hline 18 & Neg. & Neg. & Neg. & \\
\hline 19 & Neg. & Neg. & Neg. & \\
\hline 20 & Neg. & Neg. & Neg. & \\
\hline 21 & Str. pyogenes $>10^{5}$ & Str. pyogenes $>10^{5}$ & Str. pyogenes $>10^{5}$ & $\begin{array}{l}\text { Str. pyogenes } \\
>10^{5} \text { (abscess) }\end{array}$ \\
\hline 22 & $\mathrm{Neg}$ & Neg. & Neg. & Neg. (abscess) \\
\hline 23 & Neg. & Neg. & Neg. & \\
\hline 24 & Neg. & Neg. & Neg. & \\
\hline 25 & Neg. & $\begin{array}{l}\text { K. pneumoniae }>10^{5} \text {, Nonferm. } \\
\text { G-rods }>10^{5}\end{array}$ & Neg. & \\
\hline 26 & Neg. & neg. & Neg. & \\
\hline 27 & E. coli $>10^{5}$ & E.coli $>10^{5}$ & Neg. & \\
\hline 28 & Neg. & E.coli $>10^{5}$ & Neg. & \\
\hline 29 & $\mathrm{Neg}$ & Neg. & Neg. & \\
\hline
\end{tabular}

Neg.- negative cultivation, E. coli-Escherichia coli, K. pneumoniae - Klebsiella pneumoniae

Nonferm. G-rods - non-fermentable G-rods, P. aeruginosa - Pseudomonas aeruginosa, Gp.- group sp.- species, Str.- Streptococcus

In our study, we identified 4 pathogens altogether. Unlike Krawiec and Heflin (1992), reporting E. coli as by far the most frequent agent found in the prostatic fluid in $70 \%$, we did not observe any other such strong predominance of the pathogen. After cultivation of urine, some quite interesting results were disclosed. In 6 patients (out of 13), the pathogen from the prostatic fluid was identified, with negative urine cultivation, and, moreover, in patients 9 and 10, different pathogens from prostatic fluid and urine were isolated, suggesting that the prostate can be easily invaded by different bacteria than the urinary bladder. In light of this knowledge we postulate that cultivation of the urine alone is insufficient as a diagnostic procedure for prostatic disorders.

Cultivation of FNAB samples was positive in patient number 21 and 11. In patient 21, Streptococcus pyogenes was isolated from the fine needle aspirate, as well as from the abscess cavity. The difference between cultivation of the prostatic fluid and the fine needle aspirate is arguably the most appealing result of our study. Despite some other 
authors (Thrall et al. 1985; Ling et al. 1990) reporting the fine needle aspirate to be able to disclose the pathogen, in our study the accordance of FNAB and PM was achieved in two cases only. The difference was confirmed as statistically highly significant. We feel strongly that this may be a good reason to use prostatic catheter biopsy as the first-choice method for primary pathogen identification. The discrepancy can be explained by the small amount of tissue obtained by FNAB, by rather random sample obtaining, and by the fact that prostatic massage stimulates the discharge of the fluid from the whole gland, which makes the sample more practical to use for examination. Possible contamination of the fluid from the urinary bladder or urethra does not seem to be a problem, as the same pathogen in the prostatic fluid as in urine was isolated only in 4 cases, while in two other cases different pathogens in the prostatic fluid and urine were identified. In the rest of the cases, urine was found sterile, even though the prostatic fluid revealed a pathogen quite clearly. This is another evidence that the isolated pathogen originates certainly from the prostate gland. Ling et al. (1990) reported accordance in cultivation of the prostatic fluid with direct glandular tissue cultivation in 80 to $100 \%$.

By ultrasonography we used and appreciated the predictive formula set by Ruel et al.(1996) for quantification of prostatic enlargement. However, though otherwise useful, ultrasound seems to be rather too rough to establish a definite diagnosis. Difficulties are apparent especially in differentiating between BPH and chronic prostatitis. Moreover, according to Lamb (1990), the prodromal stage of BPH is ultrasonographically unrecognizable from healthy glandular tissue. On the other hand, early identification of prostatic cavities and, sometimes, differentiation of cysts and abscesses may be helpful. Whenever needed, USGguided aspiration of cavity, and evaluation of aspirate is recommended (Luersen 1993; Prüfer et al. 2000). In our experience, prostatic catheter biopsy revealed better results than the prostatic lavage advocated by some authors. As a major advantage we consider collecting of the fluid immediately after its discharge into the urethra, so contamination can be almost avoided. Compared to FNAB, a much larger amount of fluid directly from the gland can be taken as an advantage. Only in patients with general fibrosis of glandular tissue, some problems can be experienced with fluid obtaining.

Powe (2004) considers cytology as a more sensitive method than histopathology in patients with bacterial prostatitis, especially for easier identification of etiologic agents. Although Thrall et al. (1985) describe up to $96 \%$ of accordance between prostatic histopathology and cytology, in our study, only $82.76 \%$ of results of cytology (after both FNAB and CB) came back from histopathology with the same final diagnosis, while accordance between samples obtained by FNAB and those collected by CB was as low as $75.86 \%$. Extremely difficult seems to be the differentiation between chronic prostatitis and BPH. In our study we found a high rate of false negative results for both FNAB ( $p<0.05$, statistically significant), and CB ( $p<0.009$, statistically highly significant). Even though some authors give high credit to FNAB, reporting sensitivity up to $80 \%$ (Barsanti et al. 1983; Nickel and Teske 1992; Powe 2004) compared to histopathology, in our study we found the accordance of FNAB cytology with histopathology just about $72 \%$, with a small but non-significant increase after catheter biopsy. We feel that prostatic catheter biopsy gives more volume of the directly collected prostatic fluid, as well as a better reproducible sample for examination. Moreover, it provides better reproducibility of bacterial cultivation. Fine needle aspiration is essential in differentiation of cysts from abscesses and vice-versa.

In summary, prostatic catheter biopsy is often more acceptable for the owner, it is less painful and there is no risk of peritonitis during the procedure (unlike during FNAB-moving needle just withdrawn from inflammated tissue). Manual ejaculation, recommended by some authors (Kay et al. 1989) is another method of collecting the prostatic fluid; however, this is less tolerated by patients suffering from prostatic pain. The success rate of such 
method in patients with prostatitis is around 30\% (Barsanti and Finco 1989; Kay et al. 1989).

The goal of our study was to explore prostatic catheter biopsy $(\mathrm{CB})$ as a valid diagnostic procedure. We tried to compare it with other well-established methods, namely rectal palpation, ultrasonography and USG-guided fine needle aspiration biopsy (USG-FNAB). Histopathological examination was used as a confirmative. In the end we succeeded to prove that CB is no worse that USG-FNAB in establishing final diagnosis. Both methods appear less sensitive (highly significantly) in confirmation of chronic prostatitis. Moreover, in our settings, CB was clearly superior (highly significantly) to USG-FNAB in obtaining samples for bacterial cultivation and final pathogen isolation. Early diagnosis, along with a precise choice of antibiotic treatment is very important for the control of most prostatic disorders.

\section{Srovnání prostatické masáže u psa s dalšími diagnostickými metodami}

V této práci bylo použito 29 vybraných pacientů různých plemen psů s přiznaky onemocnění prostaty. U pacientů jsme provedli prepubickou cystocentézu (CC), rektoabdominální/abdominální masáž prostaty $(\mathrm{PM})$, tenkojehelnou aspirační biopsii prostaty pod USG kontrolou (FNAB) a transkutální biopsii prostaty Tru-cut jehlou pod USG kontrolou. Vzorky jsme hodnotili kultivačně, cytologicky a histopatologicky. Histopatologický nález jsme považovali za konfirmační. Po zhodnocení jednotlivých postupů jsme došli k závěru, že výpovědní hodnota prostatické masáže (PM 75,86 \%) v prresnosti cytologické diagnostiky je srovnatelná s FNAB $(72,41 \%)$. Velkým př́nosem se PM oproti FNAB zdá být $\mathrm{v}$ kultivačním vyšetření. Z prostatitické tekutiny byly patogeny vykultivovány ve 13 př́padech z 29, zatímco z FNAB pouze ve 2, což představuje ve srovnání s prostatickou masáží pouze $15 \%$ úspěšnost.

\section{References}

ATILOLA MAO, PENNOCK PW 1986: Cystic uterus masculinus in the dog. Six cases history reports. Vet Radiol Ultrasound 27: 8-14

BAKER RH, LUMSDEN JH 1999: Reproductive tract. In: BAKER RH, LUMSDEN JH (Eds): Color Atlas of Cytology of the Dog and Cat. CV Mosby, St. Louis, pp. 235-251

BARSANTI JA, FINCO DR 1989: Canine prostatic diseases. In: ETTINGER SJ, FELDMAN EC (Eds): Textbook of Veterinary Internal Medicine. Philadelphia, WB Sounders, pp. 1859-1880

BARSANTI JA, PRASSE KW, CROWELL WA, SHOTTS EB, FINCO DR 1980: Evaluation of diagnostic techniques for canine prostate disease. J Am Vet Med Assoc 177: 160-163

BELL FW, KLAUSNER JS, HAYDEN DW, FEENEY DA, JOHNSTON SD 1991: Clinical and pathologic features of prostatic adenocarcinomas in sexually intact and castrated dogs: 31 cases (1970-1987). J Am Anim Hosp Assoc 199: 1623-1630

DORFMAN M, BARSANTI JA 1995: CVT Update: Treatment of canine bacterial prostatitis. In: BONAGURA JD, KIRK RW (Eds): Current Veterinary Therapy XII. Philadelphia, WB Sounders, pp. 1029-1032

ENGLAND GC, ALLEN WE, MIDDLETON DJ 1990: An investigation into the origin of the first fraction of canine ejaculate. Res Vet Sci 49: 66-70

FEENEY DA, JOHNSTON GR, KLAUSNER JS, PERMAN V, LEININGER JR, TOMLINSON MJ 1987: Canine prostatic disease-comparsion of ultrasonographic appearance with morphologic and microbiologic findings: 30 cases (1981-1985). J Am Vet Med Assoc 190: 1027-1034

KAY ND, LING GV, NYLAND TG 1989: Cytological diagnosis of canine prostatic disease using a urethral brush technique. J Am Anim Hosp Assoc 25: 517-526

KRAWIEC DR 1994: Canine prostate disease. J Am Anim Hosp Assoc 204: 1561-1564

KRAWIEC DR, HEFLIN D 1992: Study of prostatic disease in dogs: 177 cases (1981-1986). J Am Anim Hosp Assoc 200: 1119-1122

LAMB CR 1990: Abdominal ultrasonography in small animals: intestinal tract and mesentery, kidneys, adrenal glands, uterus and prostate. J Small Anim Pract 31: 295-304

LING GV, NYLAND TG, KENNEDY PC, HAGER DA, JOHNSON DL 1990: Comparsion of two sample collection methods for quantitative bacteriologic culture of canine prostatic fluid. J Am Vet Med Assoc 196: 1479-1482

LOWSETH LA, GERLACH RF, GILLETT NA, MUGGENBURG BA 1990: Age-related changes in the prostate and testes of the beagle dog. Vet Clin Pathol 27: 347-353 
LÜERSSEN D 1993: Perkutane Drainage von Prostataabszessen unter sonographischer Dartstellung anhang von zwei Fallbeispielen. Tierarztl Prax 38: 15-20

NIELSEN SW, KENNEDY PC 1990: Tumors of the Genital System. In: MOULTON JE (Ed.): Tumors of Domestic Animals, $3^{\text {rd }}$ ed. University of California Press, Berkeley, pp. 569-570

PETER AT, STEINER JM, ADAMS LG 1995: Diagnosis and medical management of prostate disease in the dog. Semin Vet Med Surg Small Anim 10: $35-42$

PRÜFER A, LÜERSSEN D, JANTHUR M 2000: Prostate gland. In: NAUTRUP CP, TOBIAS R, CARTEE RE (Eds.): Diagnostic Ultrasonography of the Dog and Cat. London, Manson Publishing Ltd, pp. 285-289

READ RA, BRYDEN S 1995: Urethral bleeding as a presenting sign of benign prostatic hyperplasia in the dog: A retrospective study (1979 - 1993). J Am Anim Hosp Assoc 31: 261-267

REBAR AR 1987: Diagnostic cytology in veterinary practice. In: KIRK, R.W. (Ed.) Current Veterinary Therapy VII. Philadelphia, W.B. Saunders, pp. 16-27

RUEL Y, BARTHEZ PY, MAILLES A, BEGON D 1997: Ultrasonographic evaluation of the prostate in healthy intact dogs. Vet Radiol Ultrasound 39: 212-216

THRALL MA, OLSON PN, FREEMAYER EG 1985: Cytologic diagnosis of canine prostatic disease. J Am Anim Hosp Assoc 21: 95-102

WHITE RAS 2000: Prostatic surgery in the dog. Clin Tech Small Anim Pract 15: 46-51 